\title{
Multispectral phase shifting interferometry algorithm
}

\author{
Michal Wengierow*, Leszek Salbut, and Maciej Wielgus \\ ${ }^{1}$ Institute of Micromechanics and Photonics, Warsaw University of Technology, 8 Sw. A. Boboli St., 02-525 \\ Warsaw, Poland
}

Received May 15, 2012; accepted June 09, 2012; published June 30, 2012

\begin{abstract}
Standard phase shifting interferometry (PSI) algorithms are used to obtain a phase map from fringe patterns. Their spectral properties can by analyzed using frequency transform function (FTF). In this paper a new multispectral phase shifting interferometry algorithm (mPSI) has been proposed. It is a combination of two simpler PSI algorithms and enables filtering out unwanted high frequency fringes from the image. It can be used to the increase measurement range of standard interferometry by analyzing the fringe pattern created by multiplication of two other fringe patterns recorded with the use of two different wavelengths. Numerical simulations of the mPSI algorithm and the results from the real setup are shown in the paper.
\end{abstract}

Phase shifting interferometry (PSI) [1-2] algorithms are well known and very accurate techniques in optical testing. However, when a high step bigger than N/4 (in classical Twyman-Green setup) exists in a measured object there is no simple solution to obtain a correct phase map. To overcome this problem white light interferometry [3-4] can be used. Unfortunately, it is complicated and requires scanning an object in depth. Much simpler are multiwavelength techniques using fringe patterns and phase maps obtained by means of light sources with two or more different wavelengths [5-6]. In such a case the equivalent wavelength is calculated as:

$$
\lambda_{\text {eqv }}=\frac{\lambda_{1} \lambda_{2}}{\left|\lambda_{1}-\lambda_{2}\right|}
$$

There are several phase map calculating and error compensating algorithms for $\lambda_{\text {eqv }}$ [7-8]. In this paper a new approach, using a multispectral phase shifting interferometry (mPSI) algorithm is presented. It is based on standard PSI algorithms and uses their ability to filter out unwanted harmonics [9].

The fringe patterns obtained using different wavelengths can be written as:

$$
I_{n}(x, y)=a_{n}(x, y)+b_{n}(x, y) \cos \left[\frac{2 \pi}{\lambda_{n}} L(x, y)\right],
$$

where $a_{n}(x, y)$ is the background coefficient, $b_{n}(x, y)$ is the contrast modulation coefficient and $L(x, y)$ is the shape function of a measured object. Assuming that fringe patterns are normalized and omitting spatial dependence for clarity, for two different wavelengths a new fringe pattern can be calculated as a result of multiplying two single fringe patterns:

$$
\begin{aligned}
& I_{r}=I_{1} I_{2}-\left(I_{1}+I_{2}\right)= \\
& a_{r}+b_{r} \cos \left(2 \pi \frac{\left|\lambda_{1}-\lambda_{2}\right|}{\lambda_{1} \lambda_{2}} L\right)+b_{r} \cos \left(2 \pi \frac{\lambda_{1}+\lambda_{2}}{\lambda_{1} \lambda_{2}} L\right),
\end{aligned}
$$

where $a_{r}$ is the new background coefficient and $b_{r}$ is the new contrast coefficient. In the fringe pattern described as in Eq. 3 there exist two separate fringe frequencies. They correspond to two equivalent wavelengths: $\lambda_{\text {eqv- }}=\left(\lambda_{1} \lambda_{2}\right) /\left|\lambda_{1}-\lambda_{2}\right|$ and $\lambda_{\text {eqv }}=\left(\lambda_{1} \lambda_{2}\right) /\left(\lambda_{1}+\lambda_{2}\right)$. Adding temporal carrier $\omega_{0}$, Eq. 3 can be written as a complex function:

$$
\begin{aligned}
& I_{r}(t)=a_{r}+\frac{b_{r}}{2} \exp \left\{-i\left[\phi\left(\lambda_{\text {eqv }}\right)+\omega_{0} t\right]\right\}+ \\
& \frac{b_{r}}{2} \exp \left\{i\left[\phi\left(\lambda_{\text {eqv }}\right)+\omega_{0} t\right]\right\}+\frac{b_{r}}{2} \exp \left\{-i\left[\phi\left(\lambda_{\text {eqv+ }}\right)+\kappa \omega_{0} t\right]\right\}+ \\
& \frac{b_{r}}{2} \exp \left\{i\left[\phi\left(\lambda_{\text {eqv+ }}\right)+\kappa \omega_{0} t\right]\right\},
\end{aligned}
$$

where $\kappa=\lambda_{\text {eqv }} / \lambda_{\text {eqv+ }}, \omega_{0}=2 \pi c / \lambda_{\text {eqv- }}, c$ is the speed of light, $\varphi\left(\lambda_{\text {eqv }}\right)$ and $\varphi\left(\lambda_{\text {eqv+ }}\right)$ are phase functions. The aim of the mPSI algorithm is to filter out $a_{r}$, components dependent on $\lambda_{\text {eqv }}$ and one complex exponential component dependent on $\lambda_{\text {eqv }}$. The desired output signal can be written as:

$$
\begin{aligned}
& I_{c}(t)=H_{m}\left(-\omega_{0}\right) \frac{b_{r}}{2} \exp \left\{-i\left[\phi\left(\lambda_{\text {eqv- }}\right)+\omega_{0} t\right]\right\}= \\
& h_{m}(t) * I_{r}(t),
\end{aligned}
$$

where $h_{m}(t)$ is the filter's impulse response and $H_{m}(\omega)=F\left[h_{m}(t)\right]$ is its Fourier transform [10]. The minimum requirements for frequency transform function $H_{m}(\omega)$ to obtain $I_{c}(t)$ can be written as:

$$
\begin{aligned}
& H_{m}\left(-\omega_{0}\right) \neq 0, \\
& H_{m}\left(\omega_{0}\right)=H_{m}\left(-\omega_{0} \frac{\lambda_{\text {eqv- }}}{\lambda_{\text {eqv }}}\right)=H_{m}\left(\omega_{0} \frac{\lambda_{\text {eqv- }}}{\lambda_{\text {eqv }}}\right)=0 .
\end{aligned}
$$

\footnotetext{
*E-mail: m.wengierow@mchtr.pw.edu.pl
} 
The mathematical representation of a simple PSI algorithm is a linear quadrature digital filter.

$$
h(t)=\exp \left(i \omega_{o} t\right) \sum_{n} a_{n} \delta(t-n T),
$$

where $a_{n}$ is the constant coefficient, $\delta$ is the Dirac distribution and $\mathrm{T}$ is the sampling rate. Then the phase obtained from the fringe patterns can be represented as:

$$
\tan (\phi)=\left.\frac{h i(t) * I_{r}(t)}{h r(t) * I_{r}(t)}\right|_{t=0},
$$

where $h i(t)$ and $h r(t)$ are the imaginary and real components of $h(t)$. The easiest way to fulfil Eq. (6) is to combine two different filters. The first one has to be able to filter out one of complex exponential component dependent on $\lambda_{\text {eqv }}$ and the background. In addition it has to be as much insensitive to phase step miscalibration as possibile. The PSI algorithm which meets these criteria is an eight step algorithm with a bell-shaped window [2].

$$
\begin{aligned}
& h_{1}(t)=\delta(t)-11 \delta\left(t-\frac{\pi}{\omega_{0}}\right)+15 \delta\left(t-\frac{2 \pi}{\omega_{0}}\right)-5 \delta\left(t-\frac{3 \pi}{\omega_{0}}\right)+ \\
& i\left\{5 \delta\left(t-\frac{\pi}{2 \omega_{0}}\right)-15 \delta\left(t-\frac{3 \pi}{2 \omega_{0}}\right)+11 \delta\left(t-\frac{5 \pi}{2 \omega_{0}}\right)-\delta\left(t-\frac{7 \pi}{2 \omega_{0}}\right)\right\} .
\end{aligned}
$$

The second filter has to filter out the components dependent on $\lambda_{\text {eqv+. }}$. However, a narrow filter would be sensitive to wavelength instability. To meet these conditions the filter with a cosinusoidal shape of frequency transform function centred around $\omega=0$ has been proposed.

$$
h_{2}(t)=\delta(t)+2 \delta\left(t-\frac{\pi}{\omega_{0} \kappa}\right)+\delta\left(t-\frac{2 \pi}{\omega_{0} \kappa}\right) .
$$

Convolution of $h_{1}(t)$ and $h_{2}(t)$ gives the wanted filter $h_{m}(t)$.

$$
h_{m}(t)=h_{1}(t) * h_{2}(t)=\sum_{n}\left[c_{n} \delta\left(t-\alpha_{n}\right)+i\left\{s_{n} \delta\left(t-\alpha_{n}\right)\right\}\right],
$$

where $c$ is the vector of real part constants, $s$ is the vector of imaginary part constants and $\boldsymbol{\alpha}$ is the sampling rate vector corresponding to phase steps.

$$
\begin{aligned}
& c=[1,2,1,0,0,0,-11,-22,-11,0,0,0,15,30, \\
& 15,0,0,0,-5,-10,-5,0,0,0], \\
& s=[0,0,0,5,10,5,0,0,0,-15,-30,-15,0,0, \\
& 0,11,22,11,0,0,0,-1,-2,-1],
\end{aligned}
$$

$$
\begin{aligned}
& \alpha=\left[0, \frac{\pi}{\omega_{0} \kappa}, \frac{2 \pi}{\omega_{0} \kappa}, \frac{\pi}{2 \omega_{0}}, \frac{\pi}{2 \omega_{0}}+\frac{\pi}{\omega_{0} \kappa},\right. \\
& \left.\frac{\pi}{2 \omega_{0}}+\frac{2 \pi}{\omega_{0} \kappa}, \ldots, \frac{7 \pi}{2 \omega_{0}}, \frac{7 \pi}{2 \omega_{0}}+\frac{\pi}{\omega_{0} \kappa}, \frac{7 \pi}{2 \omega_{0}}+\frac{2 \pi}{\omega_{0} \kappa}\right] .
\end{aligned}
$$

As can be easily noticed, the shape of $H_{m}(\omega)$ directly depends on used wavelengths and the mPSI algorithm should be adjusted whenever they are changed. Exemplary graphs of $H_{l}(\omega), H_{2}(\omega)$ and $H_{m}(\omega)$ are shown in Figs. 1-2. Functions $H_{2}(\omega)$ and $H_{m}(\omega)$ are drawn for the following parameters: $\kappa=13.157, \lambda_{1}=632.8 \mathrm{~nm}, \lambda_{2}=543.4 \mathrm{~nm}, \lambda_{\text {eqv }}$ $=3846.3 \mathrm{~nm}, \lambda_{\text {eqv+ }}=292.3 \mathrm{~nm}$.

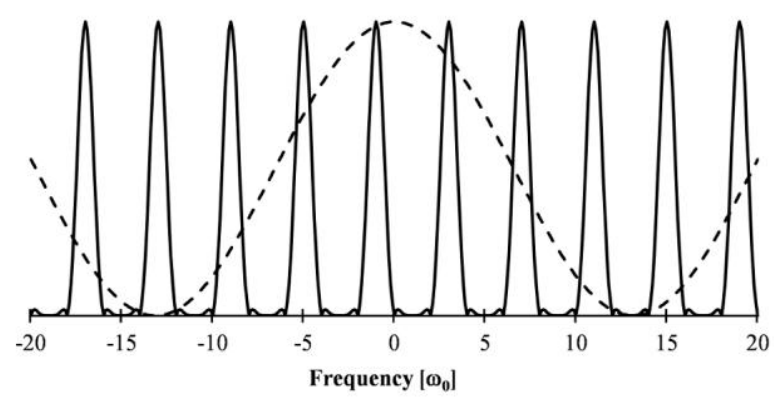

Fig. 1. Frequency transform functions of an eight step algorithm with a bell-shaped window (solid line) and cosinusoidal filter for $\kappa=13.157$ (dotted line)

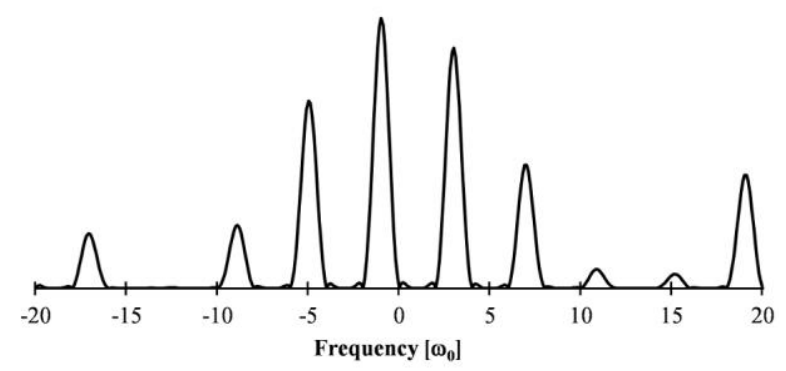

Fig. 2. Frequency transform functions of mPSI algorithm designed for $\lambda_{1}=632.8 \mathrm{~nm}, \lambda_{2}=543.4 \mathrm{~nm}$.

To confirm the correct work of the proposed mPSI algorithm and its ability to extend the measurement range a numerical test was performed. The simulated object was a flat surface with 3 steps with a height equal to $300 \mathrm{~nm}$, $900 \mathrm{~nm}$ and $1500 \mathrm{~nm}$. A $3 \mathrm{D}$ view of this object is shown in Fig. 3. The fringe patterns for $\lambda_{1}=632.8 \mathrm{~nm}$ and $\lambda_{2}=543.4 \mathrm{~nm}$ were numerically created. Then two phase maps were calculated using an eight step PSI algorithm with a bell-shaped window for $\lambda_{1}$ and $\lambda_{2}$. A third phase map was obtained using mPSI algorithms. Cross sections of phase maps after scaling to nanometers are shown in Fig 4. 


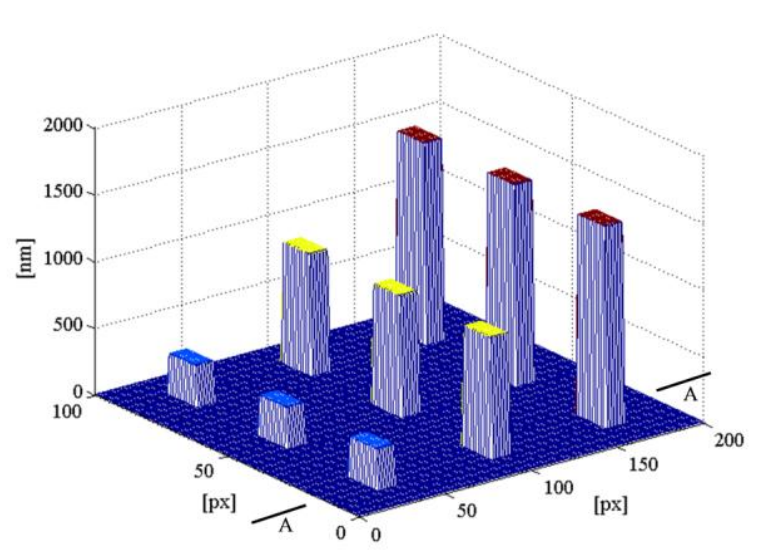

Fig. 3. Simulated surface with height steps $300 \mathrm{~nm}, 900 \mathrm{~nm}$ and $1500 \mathrm{~nm}$.

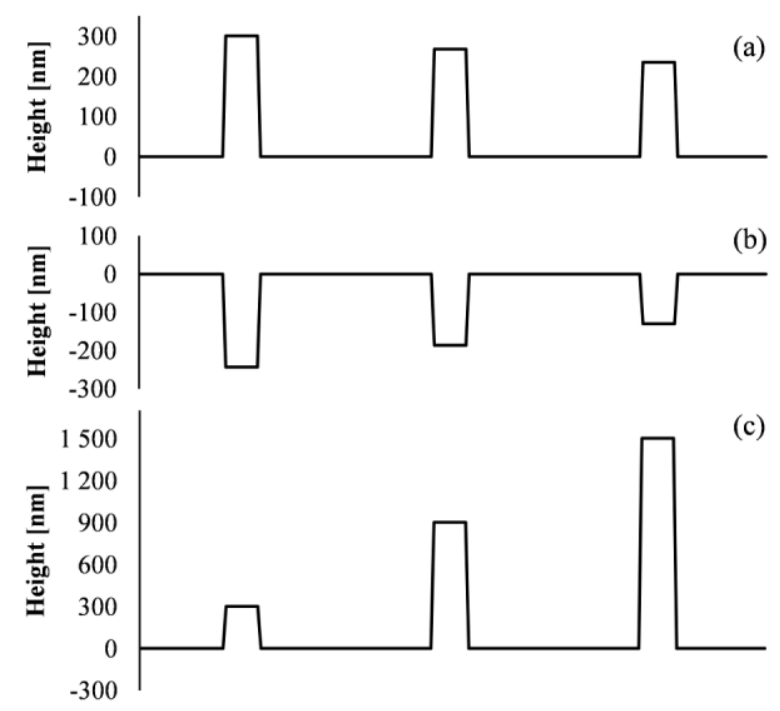

Fig. 4. Cross section A-A obtained for $\lambda_{1}=632.8 \mathrm{~nm}$ (a), $\lambda_{2}=543.4 \mathrm{~nm}$ (b) and $\lambda_{\text {eqv- }}=3846.3 \mathrm{~nm}$ (c).

It is clearly visible that an mPSI algorithm is able to extend the measurement range to $\lambda_{\text {eqv- }}=3846.3 \mathrm{~nm}$ while using only one wavelength is insufficient to measure such discontinuities properly.

A real condition test of the mPSI algorithm was carried out in the Twyman Green setup using $\lambda_{\text {red }}=632.822209 \mathrm{~nm}$ and $\lambda_{\text {green }}=543.369518 \mathrm{~nm}$. The tested object was a surface of $1 \mathrm{~m}$ gauge block wrung to platen. Phase maps were obtained using an eight step PSI algorithm with a bell-shaped window. Then they were used to create numerically normalized fringe patterns for the mPSI algorithm as in Eq. (3). An exemplary fringe pattern is shown in Fig. 5.

To compare results phase maps for mPSI algorithm and for $\lambda_{\text {red }}$ were unwrapped and scaled to nanometres. Then their differences were calculated. Exemplary cross section over difference on gauge block surface after plane fitting is shown in Fig. 6.

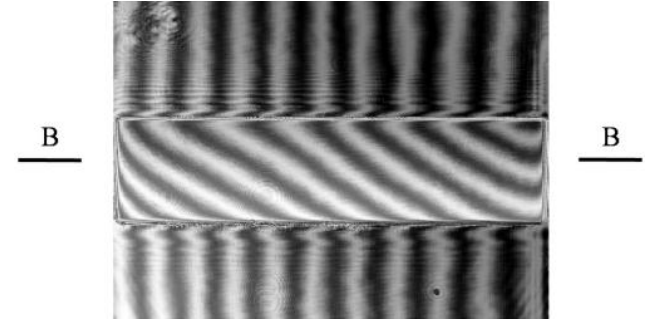

Fig. 5. Fringe pattern of gauge block wrung to platen obtained from Eq. (3).

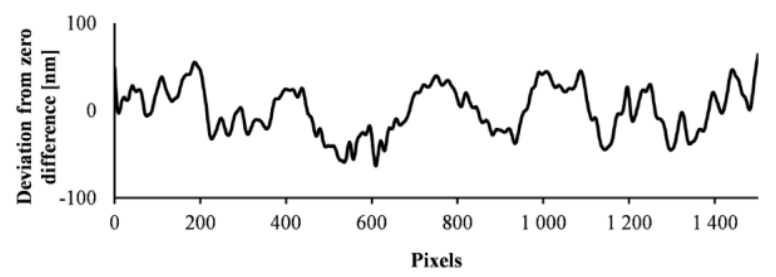

Fig. 6. Cross section B-B over difference of results obtained on gauge block surface.

The standard deviation from zero difference on gauge block surface was $33 \mathrm{~nm}$, which corresponds to less than $1 / 100$ of $\lambda_{\text {eqv. }}$. This is enough to use an mPSI phase map to unwrap phase maps obtained using a single wavelength and thereby achieve good accuracy and a wide measurement range. Moreover, the deviations were probably caused by background fluctuation due to speckles time averaging realized by a fibre shaking device in the interferometer setup [11]. It is noteworthy that an mPSI algorithm can be used to combine more than two wavelengths to measure very large discontinuities.

The work was supported within the Faculty of Mechatronics Dean's grant 504 M 11433019000 and statutory work realized in IMiF WUT.

\section{References}

[1] J. Schmit, K. Creath Appl. Opt. 34(19), 3610 (1995).

[2] J. Schmit, K. Creath Appl. Opt. 35(28), 5642 (1996).

[3] B.S. Lee, T.C. Strand Appl. Opt. 29(26), 3784 (1990).

[4] P. Hariharan, M. Roy J. Mod. Opt. 41(11), 2197 (1994).

[5] H. van Brug, Pure Applied. Opt. 7,1465 (1998).

[6] J.C. Wyant Optical Testing: Extended Range Two-Wavelength Interferometry (Optical Sciences Center University of Arizona).

[7] J. Schmit, P. Hariharan, Opt. Eng. 45, 115602 (2006).

[8] Y. Cheng, J.C. Wyant, Appl. Opt. 23(24), 4539 (1984).

[9] P. de Groot Appl. Opt. 39(16), 2658 (2000).

[10] M. Servin, J.C. Estrada, J.A. Quiroga Opt. Expr. 17(24), 21867 (2009).

[11] M. Wengierow, L. Sałbut, Z. Ramotowski Proc. SPIE 8082, 80822R (2011). 